\title{
Oleuropein as a novel anti-diabetic nutraceutical. An overview
}

\author{
Giuseppe Annunziata ${ }^{1 *}$, Maria Maisto ${ }^{1}$, Connie Schisano ${ }^{1}$, Luigi Barrea ${ }^{2}$, Roberto Ciampaglia $^{1}$, Viviana Narciso $^{1}$, \\ Gian Carlo Tenore $^{1}$ and Ettore Novellino ${ }^{1}$ \\ ${ }^{1}$ Department of Pharmacy, University of Naples Federico II, Naples, Italy \\ ${ }^{2}$ Department of Clinical Medicine and Surgery, Federico II University Medical School of Naples, Italy
}

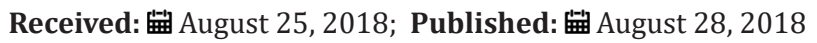

*Corresponding author: Giuseppe Annunziata, Department of Pharmacy, University of Naples Federico II, Via Domenico Montesano 49, 80131, Naples, Italy

\begin{abstract}
Among the cardiometabolic diseases, type 2 diabetes mellitus (T2DM) is one of the most diffused worldwide. Pharmacological treatments commonly used present several side effects, and this is driving people to search natural approaches. Olea europaea is currently investigated for its anti-diabetic potential. In particular, oleuropein (OLE), its main bioactive compound, have been demonstrated to exert interesting activities in management of T2DM, including hypoglycemic and antioxidant activities. The purpose of this manuscript is to review the available scientific evidence evaluating the role of OLE in managing T2DM.
\end{abstract}

Keywords: Oleuropein; Type 2 diabetes; Glucose Homeostasis; Oxidative Stress; Antioxidant

Abbreviations: T2DM: Type 2 Diabetes Mellitus; CVD: Cardiovascular Disease; AMPK: Amp- Activated Protein Kinase; OLE: Oleuropein; SGLT1: Sodium-Dependent Glucose Transporter; EFSA: European Food Safety Authority; IGT: Impaired Glucose Tolerance: TSOD: Tsumura Suzuki Obese Diabetes; GLUT: Glucose Transporter; ROS: Reactive Oxygen Species; RNS: Reactive Nitrogen Species; MDA: Malondialdehyde; HT: Hydroxytyrosol

\section{Introduction}

Among the cardiometabolic disease, type 2 Diabetes Mellitus (T2DM) is greatly diffused in the World, affecting several million people. In last few years, indeed, T2DM was considered as an epidemic [1]. At the base of the T2DM a etiology there is a reduction in $\beta$-cells function and/or the presence of insulin-resistance (IR), causing chronically elevated glycemic levels [2,3]. Chronic hyperglycemia, in turn, is one of the main causes of oxidative stress, which is recognized to be the principal responsible for T2DM complications [4-7].

Currently, for treatment of T2DM have been developed standard pharmacological treatments which, however, present several side effects, and this is driving people to search natural approaches [8]. The use of natural approaches for the treatment of metabolic diseases, indeed, is currently the most discussed issue, involving public opinion, researcher and physician. Among the main points in favor of the diffusion of natural remedies in the clinical practice, surely emerge the almost total absence of side effects, contraindications and/or drug-interactions, that make these products safe. On the other hand, the expensive cost of some of them and the lack in documented efficacy represent some of the main reasons for the suspicion that people still have for these products.

In this last years, indeed, the interest of the research in elucidating effects and mechanisms of natural bioactive compounds is strongly growing reflecting, in parallel, the interest of patients and pharmaceutical market. In this scenario, nutraceutical predominantly appears, representing the main approach for the treatment of borderline conditions, as add-on therapy and for the prevention of several pathologies, including obesity, cardiovascular disease (CVD) and T2DM. Numerous recent publications, indeed, are available in scientific literature, demonstrating the efficacy and safety of nutraceutical products which, sometimes, seem to exert activities comparable to those of common drugs.

T2DM is one of the main pathologies for which management, although the principal choice is represented by diet and life-style 
intervention $[9,10]$, nutraceutical plays a key role as therapeutic approach before the pharmaceutical treatment. Several foodderived bioactive compounds have been investigated for the anti-diabetic effects [11]. To give some examples, beside the wellknown effects of many Phyto complexes or bioactive compounds, polyphenols from coffee have been demonstrated to exert positive actions in treatment of T2DM and its complications [12]. Furthermore, in a recent editorial we reviewed the potential of resveratrol (the most representative polyphenols in grape) in treatment of T2DM and obesity, which acts mainly by activating the AMP-activated protein kinase (AMPK), thus, simulating the effects of some drugs, such as metformin [13].

Furthermore, tea polyphenols have been demonstrated to influence the glucose metabolism in HepG2 cell lines; in particular, white tea polyphenols exert the best activity in reducing the glucose uptake [14]. This is just to provide few examples of the innumerable nutraceutical remedies potentially useful in the treatment of this widespread pathology. Recently, the research in nutraceutical field is focusing on the evaluation of further matrix, to formulate novel nutraceutical products. Among these a growing interest is for the bioactive compounds contained in olive. In olive oil, indeed, over 30 biphenolic compounds have been identified [11], and to some of them has been attributed a promising therapeutic potential in T2DM management. The aim of the present document is to review the recent available literature about the role of oleuropein (OLE) in management of T2DM.

\section{Oleuropein: from chemistry to biology}

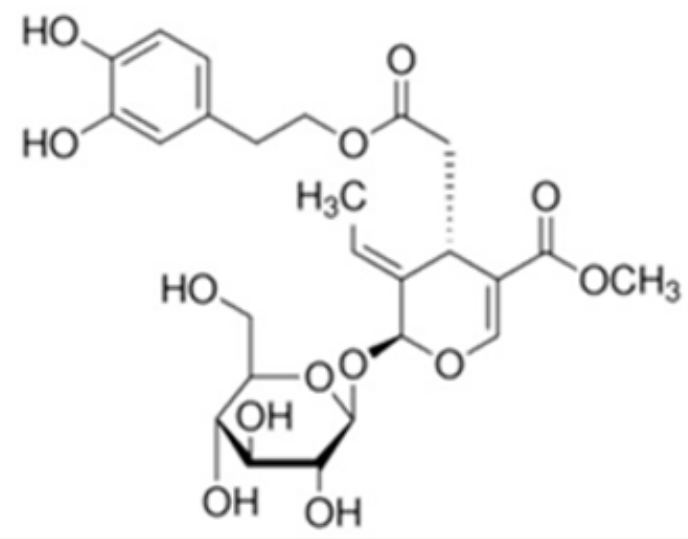

Figure 1: Chemical structure of oleuropein

Among the polyphenols contained in olives and olives-derived products, OLE (Figure 1) is the most representative, especially present in leaves. Three main portions constitute it hydroxytyrosol (HT), elenolic acid and a glucose residue $[15,16]$. This peculiar structure may be considered strongly implicated in OLE biological activities. In particular, OLE is undergoes to intestinal metabolism, with consequent release of HT or the aglycone form and resulting in increasing bioavailability and antioxidant activities [11]. On the other hand, since OLE exists in a glycoside form, it could be potentially absorbed through sodium-dependent glucose transporter (SGLT1) in the epithelial intestinal cells, thus competing with glucose, as previously reported for other glucosides [17]. This is only one of the anti-diabetic mechanisms of OLE, which will be discussed above.

Several activities were attributed to OLE and its derivatives, including hypoglycemic, cardioprotective, antioxidant, anticancer and anti-obesity activities [18]. According to the evidence regarding the beneficial effects of OLE, the European Food Safety Authority (EFSA) recommend the daily consumption of $5 \mathrm{mg} \mathrm{HT}$ and its derivatives (corresponding to $20 \mathrm{~g}$ extra virgin olive oil) to protect from CVD [19].

\section{Anti-diabetic effects of oleuropein}

Evidence regarding the anti-diabetic role of OLE are scarce. Most of the published studies, indeed, investigated the effects of leaves extracts containing OLE. In addition, different dosage was used.

\section{In vivo evidence}

Several studies were conducted on rodents models. In diabetic rats induced with streptozotocin, intraperitoneal administration of $5 \mathrm{mg} / \mathrm{kg}$ OLE significantly reduced serum glucose [20]. In other mice diabetic model OLE administration $(20,40$ or $60 \mathrm{mg} / \mathrm{kg}$ daily) significantly reduced blood glucose [21]. Murotomi et al. [22] demonstrated that the supplementation with olive leaves extract containing more that 35\% OLE significantly improved chronic hyperglycemia and impaired glucose tolerance (IGT) in Tsumura Suzuki Obese Diabetes (TSOD) mice.

Moreover, OLE was demonstrated to improve the IR (evaluated by the Homeostasis model assessment: insulin resistance, HOMAIR) [23] and serum glucose in diabetic rats induced with alloxan treated with 8 and $16 \mathrm{mg}$ OLE per $\mathrm{kg}$ body weight for 4 weeks [24]. Evidence in human demonstrated the ability of OLE to reduce glycated hemoglobin (HbA1c) [25], suggesting, thus, its efficacy in controlling the blood glucose levels. Additionally, administration of $20 \mathrm{mg}$ OLE before the meal significantly reduced blood glucose and increased serum insulin [26]. Furthermore, in middle-aged overweight men diets rich in OLE significantly improved insulin sensitivity [27].

\section{Mechanisms}

In vitro studies were conducted to elucidate the mechanisms of action of OLE in management of T2DM and provide an explication for the in vivo evidence (Figure 2). One of the first mechanisms proposed is the inhibition of several enzymes involved in glucose metabolism. OLE, indeed, was demonstrated to inhibit both rat and human intestinal maltase and human sucrase activities in a dosedependent manner [28]. 


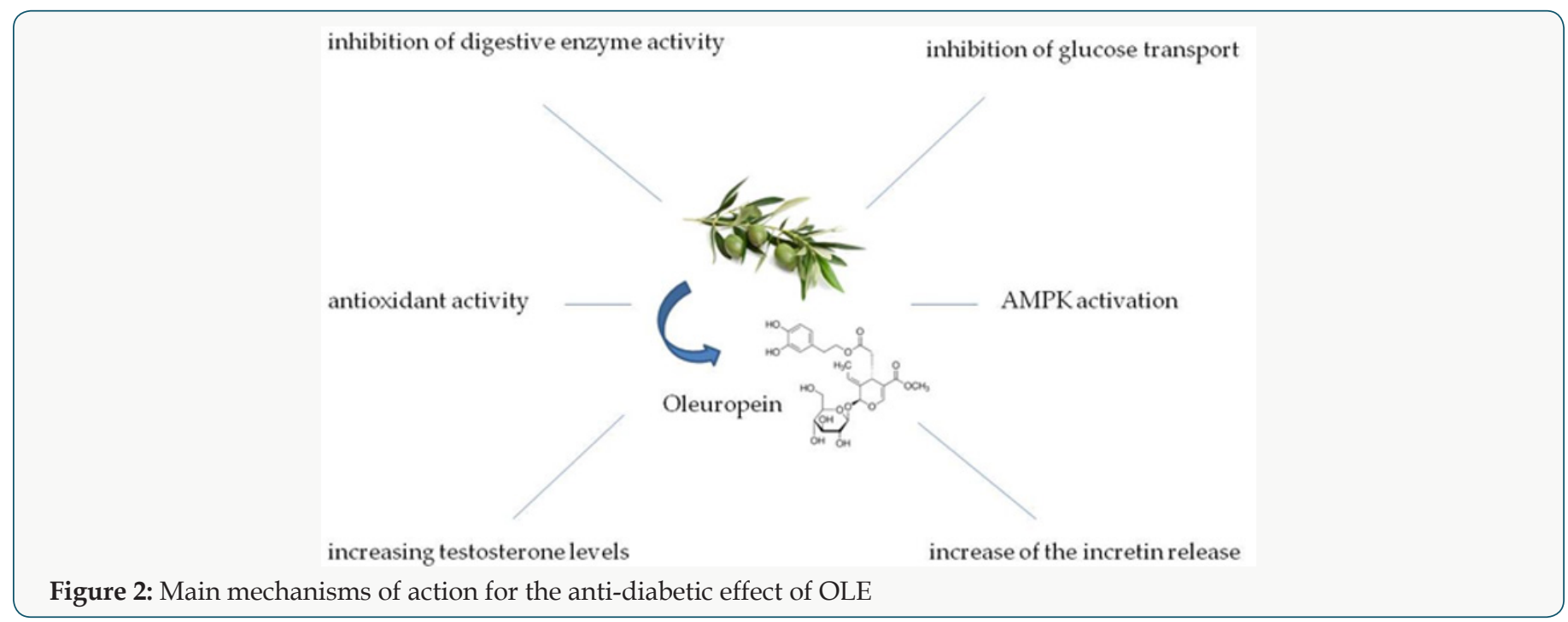

Beside the inhibition of digestive enzymes, an in vitro study performed on Caco2/TC7 cell monolayer reported that OLE can inhibit the transport of glucose with IC50 of about $0.5 \mathrm{mg}$ OLE per ml. Interestingly, it was observed that OLE inhibited in a dosedependent manner the glucose transporter (GLUT)2, but not GLUT5, which transports fructose [28]. In addition, as previously reported OLE may compete with glucose for the transport through the SGLT1, due to its peculiar structure which include a glucose residue [17].

Interestingly, in healthy subjects the administration of $20 \mathrm{mg}$ OLE significantly increased the levels of glucagon like peptide (GLP-1) compared to placebo [26], contributing to attenuate the postprandial glycaemic levels. Although it was not speculated, probably OLE may act stimulating the taste receptors in the intestine, resulting in increased the release of incretin, as reported for other phytochemicals, including hop-derived bitter compounds [29].

A recent in vitro study demonstrated that OLE activates the AMPK [30]. This is an interesting mechanism of action previously proposed to explain the anti-diabetic potential of other bioactive compounds, such as resveratrol [13]. AMPK is a signaling that regulates cellular energy metabolism [31]; in particular, it promotes the translocation of GLUT4 on the muscle cells membrane [32], thus it results involved in the peripheral glucose uptake, contributing to controlling the blood glucose levels. The activation of the AMPK pathway is the mechanism of action of several drugs commonly used for the treatment of T2DM and obesity, such as metformin $[33,34]$. The identification of novel bioactive compounds able to act by AMPK activation, thus, is useful as a novel natural approach for the management of T2DM.

Of note, the antioxidant activity was recognized as a potential and useful mechanism of action for the anti-diabetic activity of OLE, which is demonstrated to be a potent reactive oxygen species (ROS) [16] and reactive nitrogen species (RNS) scavenger
[35]. In particular, OLE has been reported to prevent the $\beta$-cells damage caused by oxidative stress [36,37], in particular by activating the Nuclear transcription factor (erythroid-derived-2)like 2 (Nrf2)/ Antioxidant response element (ARE) [38] and upregulating protective enzymes, such as the thioredoxin reductase [39]. Additionally, OLE was reported to decrease the serum malondialdehyde (MDA) and to increase the superoxide dismutase (SOD) levels in diabetic rats, which represent a partly explanation for the improvement of fasting blood glucose and glucose tolerance [40]. Moreover, in humans $20 \mathrm{mg}$ OLE is able to down-regulate the activation of Nox2 [26], that is the most ROS producer [41].

A novel interpretation for the insulin-sensitizing activity of OLE was proposed, involving the ability of OLE to increase the testosterone concentrations and plasma corticosterone and $\mathrm{LH}$ levels in rats [42]. These anabolic effects have been considered relevant in the management of T2DM, due to the role of testosterone deficiency in promoting the IR [43]. In addition, studies conducted in knockout mice made clarity on the role of the androgen receptors signaling in regulation of insulin action and homeostasis of glucose [44].

\section{Conclusion and future perspectives}

Evidence available in scientific literature showed the beneficial effects of OLE in management of T2DM. Particularly, it acts through different mechanisms of action, as shown in Figure 2, including:

a. Inhibition of digestive enzymes activities,

b. Inhibition of glucose transport,

c. Increase of the incretin release,

d. AMPK activation

e. antioxidant activity,

f. Increasing testosterone levels

However, evidence is limited, in particular in vivo human studies. Further investigation on healthy and diabetic subjects 
are needed in order to confirm the anti-diabetic potential of OLE and provide more information for the formulation of OLE-based nutraceutical products.

\section{References}

1. (2017) IDF Recommendations for managing type 2 diabetes in primary care.

2. Diagnosis and classification of diabetes mellitus (2014) Diabetes Care (37 Suppl 1): S81-S90.

3. Standards of Medical Care in Diabetes-2017 Abridged for Primary Care Providers (2017) Clinical Diabetes 35(1): 5-26.

4. Aronson D (2008) Hyperglycemia and the pathobiology of diabetic complications. Adv Cardiol 45: 1-16.

5. Baynes JW, SR Thorpe (1999) Role of oxidative stress in diabetic complications: a new perspective on an old paradigm. Diabetes 48(1): 1-9.

6. Ceriello A (2009) Oxidative stress and glycemic regulation. Metabolism 49(2 Suppl 1): 27-29.

7. Maritim AC, RA Sanders, JB Watkins 3rd (2003) Diabetes, oxidative stress, and antioxidants: a review. J Biochem Mol Toxicol 17(1): 24-38.

8. Ota A, NP Ulrih (2017) An Overview of Herbal Products and Secondary Metabolites Used for Management of Type Two Diabetes. Front Pharmacol 8: 436.

9. Knowler WC, et al. (2002) Reduction in the incidence of type 2 diabetes with lifestyle intervention or metformin. N Engl J Med 346(6): 393-403.

10. Tuomilehto J, Lindström J, Eriksson JG, Valle TT, Hämäläinen H, et al. (2001) Prevention of type 2 diabetes mellitus by changes in lifestyle among subjects with impaired glucose tolerance. N Engl J Med 344(18): 1343-1350.

11. Alkhatib A, C Tsang, J Tuomilehto (2018) Olive Oil Nutraceuticals in the Prevention and Management of Diabetes: From Molecules to Lifestyle. Int J Mol Sci 19(7).

12. Arnone A, Di Mattia E, De Rosa G, Medugno L, Annunziata G (2017) Coffee or caffeine, which has the antidiabetic effect? An overview. Piante Medicinali 16(3): 9-13.

13. Annunziata G, Tenore GC, Novellino E (2018) Resveratrol-based Nutraceuticals for the Management of Diabetes and Obesity: Real Therapeutic Potential or a mere Palliative. Archives of Diabetes \& Obesity $1(2)$.

14. Tenore GC, Stiuso P, Campiglia P, Novellino E (2013) In vitro hypoglycaemic and hypolipidemic potential of white tea polyphenols. Food Chem 141(3): 2379-2384.

15. Manna C, D’Angelo S, Migliardi V, Loffredi E, Mazzoni O, et al. (2002) Protective effect of the phenolic fraction from virgin olive oils against oxidative stress in human cells. J Agric Food Chem 50(22): 6521-6526.

16. Barbaro B, Toietta G, Maggio R, Arciello M, Tarocchi M, et al. (2014) Effects of the olive-derived polyphenol oleuropein on human health. Int J Mol Sci 15(10): 18508-1824.

17. Arts IC, AL Sesink, PC Hollman (2002) Quercetin-3-glucoside is transported by the glucose carrier SGLT1 across the brush border membrane of rat small intestine. J Nutr 132(9): 2823.

18. Lee OH, BY Lee (2010) Antioxidant and antimicrobial activities of individual and combined phenolics in Olea europaea leaf extract. Bioresour Technol 101(10): 3751-3754.

19. EFSA (2011) Scientific Opinion on the substantiation of health claims related to polyphenols in olive. EFSA Journal 9: 2033.

20. Sangi SM, Sulaiman MI, El-Wahab MF, Ahmedani EI, Ali SS (2015) Antihyperglycemic effect of thymoquinone and oleuropein, on streptozotocin-induced diabetes mellitus in experimental animals. Pharmacogn Mag 11(Suppl 2): S251-257.

21. Nekooeian AA, A Khalili, MB Khosravi (2014) Oleuropein offers cardioprotection in rats with simultaneous type 2 diabetes and renal hypertension. Indian J Pharmacol 46(4): 398-403.

22. Murotomi K, Umeno A, Yasunaga M, Shichiri M, Ishida N, et al. (2015) Oleuropein-Rich Diet Attenuates Hyperglycemia and Impaired Glucose Tolerance in Type 2 Diabetes Model Mouse. J Agric Food Chem 63(30): 6715-6722.

23. Kim SW, , Hur W, Li TZ, Lee YK, Choi JE, et al. (2014) Oleuropein prevents the progression of steatohepatitis to hepatic fibrosis induced by a highfat diet in mice. Exp Mol Med 46: e92.

24. Jemai H, A El Feki, S Sayadi (2009) Antidiabetic and antioxidant effects of hydroxytyrosol and oleuropein from olive leaves in alloxan-diabetic rats. J Agric Food Chem 57(19): 8798-8804.

25. Wainstein J, Ganz T, Boaz M, Bar Dayan Y, et al. (2012) Olive leaf extract as a hypoglycemic agent in both human diabetic subjects and in rats. J Med Food 15(7): 605-610.

26. Carnevale R, Silvestri R, Loffredo L, Novo M, Cammisotto V, et al. (2018) Oleuropein, a component of extra virgin olive oil, lowers postprandial glycaemia in healthy subjects. Br J Clin Pharmacol 84(7): 1566-1574.

27. de Bock M, José GB Derraik, Christine M Brennan, Janene B Biggs, Philip E Morgan, et al. (2013) Olive (Olea europaea L.) leaf polyphenols improve insulin sensitivity in middle-aged overweight men: a randomized, placebo-controlled, crossover trial. PLoS One 8(3): e57622.

28. Kerimi A, et al. (2018) Nutritional implications of olives and sugar: attenuation of post-prandial glucose spikes in healthy volunteers by inhibition of sucrose hydrolysis and glucose transport by oleuropein. Eur J Nutr.

29. Barrea L, Nyambe-Silavwe H, Pyner A, Oladele E, Gauer JS, et al. (2018) Could hop-derived bitter compounds improve glucose homeostasis by stimulating the secretion of GLP-1? Crit Rev Food Sci Nutr pp. 1-8.

30. Hadrich F, Garcia M, Maalej A, Moldes M, Isoda H, et al. (2016) Oleuropein activated AMPK and induced insulin sensitivity in C2C12 muscle cells. Life Sci 151: 167-173.

31. Sashidhara KV (2014) Poliothrysoside and its derivatives as novel insulin sensitizers potentially driving AMPK activation and inhibiting adipogenesis. European Journal of Medicinal Chemistry 86: 570-577.

32. Hardie DG (2011) AMP-activated protein kinase: an energy sensor that regulates all aspects of cell function. Genes Dev 25(18): 1895-1908.

33. Zhou G (2001) Role of AMP-activated protein kinase in mechanism of metformin action. J Clin Invest 108(8): 1167-1174.

34. Fryer LGD, A Parbu Patel, D Carling (2002) The anti-diabetic drugs rosiglitazone and metformin stimulate AMP-activated protein kinase through distinct signaling pathways. Journal of Biological Chemistry 277(28): 25226-25232.

35. de la Puerta R (2001) Effects of virgin olive oil phenolics on scavenging of reactive nitrogen species and upon nitrergic neurotransmission. Life Sci 69(10): 1213-1222.

36. Rigacci S, M Stefani (2016) Nutraceutical Properties of Olive Oil Polyphenols. An Itinerary from Cultured Cells through Animal Models to Humans. Int J Mol Sci 17(6).

37. Konstantinidou V (2009) Time Course of Changes in the Expression of Insulin Sensitivity-Related Genes after an Acute Load of Virgin Olive Oil. Omics-a Journal of Integrative Biology 13(5): 431-438.

38. Visioli F, G Bellomo, C Galli (1998) Free radical-scavenging properties of olive oil polyphenols. Biochemical and Biophysical Research Communications 247(1): 60-64.

39. Peng S (2015) Dual protection of hydroxytyrosol, an olive oil polyphenol, against oxidative damage in PC12 cells. Food Funct 6(6): 2091-2100. 
40. Khalili A, AA Nekooeian, MB Khosravi (2017) Oleuropein improves glucose tolerance and lipid profile in rats with simultaneous renovascular hypertension and type 2 diabetes. J Asian Nat Prod Res 19(10): 1011-1021.

41. Violi F (2017) NADPH Oxidase-2 and Atherothrombosis: Insight From Chronic Granulomatous Disease. Arterioscler Thromb Vasc Biol 37(2): 218-225.

42. Alhazza IM, Bashandy SA (2007) Hypoglycemic, hypolipidemic, antioxidant and male sexual improvement potentials of olive oil in alloxan treated rats. Journal of Pharmacology and Toxicology 2(5): 427436.

43. Kapoor D (2005) Androgens, insulin resistance and vascular disease in men. Clin Endocrinol (Oxf) 63(3): 239-250.

44. McInnes KJ (2012) Deletion of the Androgen Receptor in Adipose Tissue in Male Mice Elevates Retinol Binding Protein 4 and Reveals Independent Effects on Visceral Fat Mass and on Glucose Homeostasis. Diabetes 61(5): 1072-1081.

\section{(c) \\ This work is licensed under Creative \\ Commons Attribution 4.0 License}

To Submit Your Article Click Here:

Submit Article

DOI: 10.32474/AD0.2018.01.000113

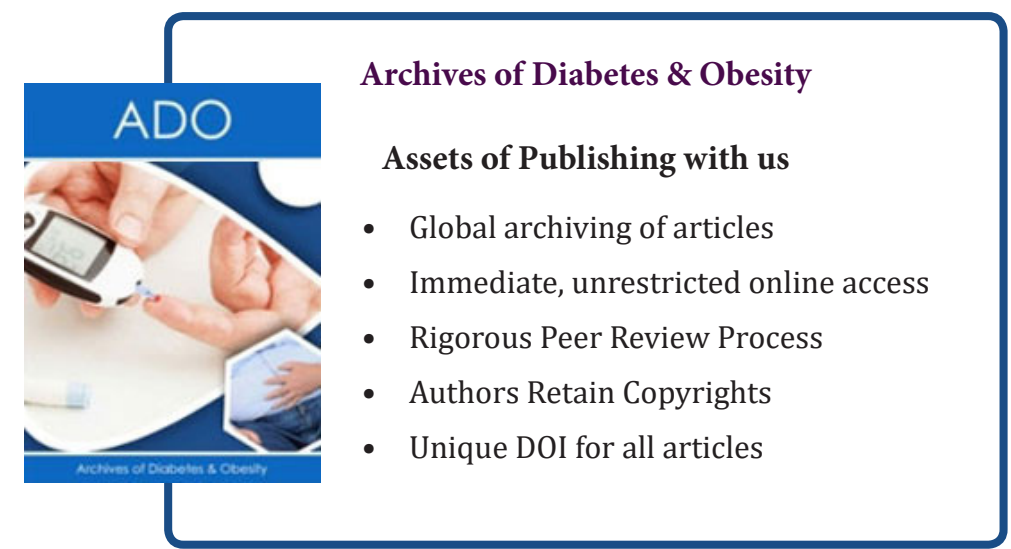

